\title{
Management of vitreous floaters: an international survey the European VitreoRetinal Society Floaters study report
}

\author{
Ece Ozdemir Zeydanli $\mathbb{1}^{1} \cdot$ Barbara Parolini $^{2}$ - Sengul Ozdek ${ }^{3}$ Silvia Bopp ${ }^{4} \cdot$ Ron A. Adelman ${ }^{5}$. Ferenc Kuhn ${ }^{6}$. \\ Giampaolo Gini ${ }^{7} \cdot$ Ahmed B. Sallam ${ }^{8} \cdot$ Nur Aksakal $^{9} \cdot$ for the EVRS Floaters Study Group
}

Received: 18 August 2019 / Accepted: 19 November 2019 / Published online: 20 April 2020

(c) The Author(s), under exclusive licence to The Royal College of Ophthalmologists 2020

\section{Learning Objectives}

Upon completion of this activity, participants will be able to:

1. Describe symptomatic improvement after PPV for symptomatic floaters, according to results from a retrospective survey study.

2. Determine safety outcomes after PPV for symptomatic floaters, according to results from a retrospective survey study.

3. Identify clinical implications of the efficacy and safety of PPV for symptomatic floaters, according to results from a retrospective survey study.

\section{Accreditation statements}

In support of improving patient care, this activity has been planned and implemented by Medscape, LLC and Springer Nature. Medscape, LLC is jointly accredited by the Accreditation Council for Continuing Medical Education (ACCME), the Accreditation Council for Pharmacy Education (ACPE), and the American Nurses Credentialing Center (ANCC), to provide continuing education for the healthcare team.

\section{Credit hours}

1.0

Release date:

Expiration date: 19 April 2020

Post-test link: https://medscape.org/eye/posttest924758

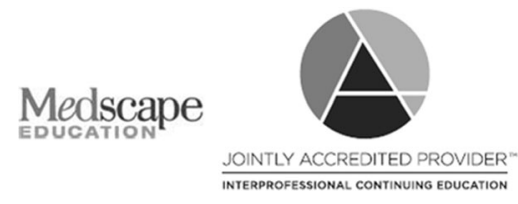

This study was presented at the 16th European Vitreo-Retinal Society Congress, 2016.

Members of the EVRS Floaters Study Group are listed at the end of paper.

Supplementary information The online version of this article (https:// doi.org/10.1038/s41433-020-0825-0) contains supplementary material, which is available to authorized users.

Ece Ozdemir Zeydanli

eceozdemirzeydanli@gmail.com

Polatlı State Hospital, 06900 Ankara, Turkey

2 EyeCare Clinic, Via Cefalonia 70, 25124 Brescia, Italy

3 Gazi University School of Medicine, 06500 Ankara, Turkey

4 Capio Augenklinik Universitätsallee, Parkallee 301, D-28213 Bremen, Germany

\section{Authors/Editors disclosure information}

Sobha Sivaprasad, MD, has disclosed the following relevant financial relationships: Served as an advisor or consultant for: Allergan, Inc.; Bayer AG; Boehringer Ingelheim Pharmaceuticals, Inc.; Heidelberg Pharma GmbH; Optos; Roche. Served as a speaker or a member of a speakers bureau for: Allergan, Inc.; Bayer AG; Novartis Pharmaceuticals Corporation; Optos. Received grants for clinical research from: Allergan, Inc.; Bayer AG; Boehringer

5 Yale University School of Medicine, New Haven, CT, USA

6 Helen Keller Foundation for Research and Education, Birmingham, AL, USA

7 Eye Unit, Southampton University Hospital, Southampton, UK

8 Jones Eye Institute, University of Arkansas for Medical Sciences, Little Rock, AR, USA

9 Department of Public Health, Gazi University School of Medicine, 06500 Ankara, Turkey 
Ingelheim Pharmaceuticals, Inc.; Novartis Pharmaceuticals Corporation; Optos. EOZ, MD, has disclosed no relevant financial relationships. BP, MD, has disclosed no relevant financial relationships. SO, MD, has disclosed no relevant financial relationships. SB, MD, has disclosed the following relevant financial relationships: served as a speaker or a member of a speakers bureau for: Allergan, Inc.; Bayer AG; Novartis Pharmaceuticals Corporation. RAA, MD, MPH, has disclosed no relevant financial relationships. FK, MD, has disclosed no relevant financial relationships. GG, MD, has disclosed no relevant financial relationships. AS, MD, has disclosed no relevant financial relationships. NA, MD, has disclosed no relevant financial relationships.

\title{
Journal CME author disclosure information
}

Laurie Barclay, MD, Freelance writer and reviewer, Medscape, LLC, and has disclosed no relevant financial relationships.

\begin{abstract}
Background/objectives To evaluate the efficacy and safety of pars plana vitrectomy for symptomatic floaters.

Subjects/methods Forty-eight vitreoretinal surgeons from 16 countries provided information on 581 eyes who underwent vitrectomy for floaters in this retrospective survey study conducted by European VitreoRetinal Society. Percentage symptomatic improvement, incidence of retinal tears/detachment and post-vitrectomy cataract surgery, and the factors associated with satisfaction and complications were investigated.

Results Ninety-two percent were satisfied with the results, with $86.3 \%$ reporting complete resolution of daily-life symptoms. Overall satisfaction was lower in patients with smaller vitreous opacities at presentation (OR:0.4). Iatrogenic retinal breaks occurred in 29 eyes (5\%). Core vitrectomy and cut rates of $1500-4000$ or $>4000$ cuts/min were associated with lower risk of retinal breaks than complete vitrectomy (OR:0.05) and cut rates $<1500$ cuts/min (OR: 0.03, 0.12, respectively). Fourteen eyes (2.4\%) developed retinal detachment at a median of 3 months; and $84(48.6 \%)$ developed cataract at a median of 16 months post-vitrectomy.

Conclusions Pars plana vitrectomy resulted in high patient satisfaction with relatively low rate of severe complications in a large group of patients. The procedure may be safer when core vitrectomy and cut rates $>1500$ cuts/min are favoured. Proper patient selection and informed consent are the most important aspects of surgery.
\end{abstract}

\section{Introduction}

Vitreous floaters are caused by degenerative or pathologic alterations in the vitreous ultrastructure and perceived as shadows or fly-like obscurations to vision [1-3]. While patients with floaters often improve over time because of peripheral displacement of vitreous opacities or cognitive adaption [4], there remain a subgroup with persistent symptoms. Physicians often underestimate how much of an impact floaters may have on patients' life; however, recent studies indicated that they can be highly debilitating [5-7]. In one study, the negative impact of the floaters was so severe that the patients were willing to accept a 7\% risk of blindness to get rid of them [5].

Two treatment options have been advocated to date: $\mathrm{Nd}$ : YAG vitreolysis and pars plana vitrectomy (PPV) [8-12]. Nd: YAG vitreolysis appears to have limited efficacy, often leaving a significant amount of residues that cause persistence of the symptoms [8]. In addition, there is a potential risk of laser damage to the retina. Conversely, PPV is curative, since vitreous opacities can be permanently removed. Although reported complication rates are relatively low, vitrectomy is invasive and development of iatrogenic retinal breaks and cataract are concerns $[9,10]$. As floater treatment is mostly patient-driven with lack of objective signs to support the indication of the surgery, it is still a debate whether the symptoms of the patients justify the potential risks.

In this study, members of the European VitreoRetinal Society (EVRS) retrospectively reported the outcome and safety for treatment of vitreous floaters. This study aimed to investigate the post-treatment patient satisfaction levels and complications of PPV for vitreous floaters in a large set of patients.

\section{Methods}

The EVRS Floaters Study was a nonrandomized, retrospective, multicentre survey initiated in the autumn of 2015. Members of the society who wish to contribute were requested to fill out the study-specific data-entry forms for each patient through a web portal created on the EVRS website. Data included patient demographics, refraction error, lens status, duration of symptoms, floater severity, surgical details (vitrectomy quality/gauge/cutting speed/combined phacoemulsification), intraoperative and postoperative complications, preoperative and postoperative discomfort levels and patient satisfaction. Floaters were graded according to the severity of ophthalmoscopically visible vitreous opacities as huge, dense, small and almost invisible. Vitrectomy quality 
was reported as "complete vitrectomy" if peripheral vitreous was totally removed along with PVD induction unless there was a preexisting PVD; "subtotal vitrectomy" if PVD was induced but vitreous base not cleared extensively; "core vitrectomy" if the surgery was limited to the removal of central vitreous without PVD induction. Cutting speed was grouped into "<1500", "1500-4000" and ">4000" cuts/min (cpm). Discomfort intensity in everyday life, in professional life and psychologically was graded as "no", "light", "moderate" and "high". Patient satisfaction after surgery was graded as "worse", "equal", "satisfied" and "extremely satisfied". Eyes with previous vitrectomy, $<6$ months of follow-up, or incomplete data were excluded. The results were analysed independently of the contributor surgeons. EVRS Ethics Committee approved the study design. Because this study involved 16 different countries and institutional review board regulations differed by location; each participant was responsible for following the specific requirements of their own institution.

\section{Statistical analysis}

Descriptive data were presented as frequencies, percentages, mean ( \pm standard deviation) and median values (minimum, maximum). For categorical variables; Pearson $\chi^{2}, \chi^{2}$ for trend, Yate's corrected $\chi^{2}$, and Fisher's exact tests were used for comparisons. For continuous data, depending on normality, Independent Samples $t$-test or Mann-Whitney $U$ test; and for correlation analysis Spearman's test was used. Multivariable logistic regression models were fitted to identify various factors that were thought to be correlated with complications and patient satisfaction. Age, sex and the factors that revealed a $p$ value of $\leq 0.20$ in univariate analyses were included in the models. Statistical analyses were performed using SPSS v22.0 and significance was set as two-tailed $p$ value $<0.05$.

\section{Results}

A total of 678 eyes (645 patients) were reported by 48 experienced vitreoretinal surgeons from 16 countries. After excluding 90 eyes with $<6$ months of follow-up and 7 eyes with missing data, 581 eyes remained eligible for analyses. Patient demographics, ocular features and surgical details are summarized in Table 1.

\section{Complications}

\section{Cataract}

Combined phacovitrectomy was performed in 91 eyes, including 2 eyes that had inadvertent lens touch during
Table 1 Patient demographics, ocular features and surgical details.

\begin{tabular}{ll}
\hline Patient age (median, range) & $65(21-94)$ years \\
Patient sex & 287 male, 281 female \\
Duration of symptoms (median, range) & $12(1-99)$ months \\
Length of follow-up (median, range) & $20(6-94)$ months \\
Baseline refractive status (median, range) & \\
$\quad$ Myopia & 181 eyes, $-3.00(-23.00$ to -1.0$)$ D \\
$\quad$ Hyperopia & 101 eyes, $+2.00(+1.00$ to +10.00$)$ D \\
Baseline lens status (\% of eyes) & \\
$\quad$ Phakic & $264(45.7)$ \\
$\quad$ Pseudophakic & $314(54.3)$ \\
Quality of vitrectomy (\% of eyes) & $165(28.4)$ \\
Complete & $288(49.6)$ \\
Subtotal & $128(22)$ \\
Core & \\
Gauge (\% of eyes) & $106(18.3)$ \\
20 & $200(34.5)$ \\
23 & $254(43.9)$ \\
25 & $19(3.3)$ \\
27 & $148(25.5)$ \\
Cutting speed (\% of eyes) & $269(46.4)$ \\
$<1500$ cuts/min & $163(28.1)$ \\
$1500-4000$ cuts/min & $91(34.5)$ \\
$>4000$ cuts/min & $35(6)$ \\
Combined phacovitrectomy (\% of phakic eyes) \\
Additional ILM peeling (\% of eyes)
\end{tabular}

surgery. Of 173 eyes that were phakic after PPV, 84 $(48.6 \%)$ developed cataract requiring surgery at a median of 16 months (range, 1-67 months) post-vitrectomy. The mean age of this subgroup was $60 \pm 12.5$ years. There was no correlation between the age at which the vitrectomy was performed and the delay of cataract formation $(r=-1.17$, $p=0.28)$. None of the potential risk factors we explored were associated with the cataract formation at multivariable analysis (Table 2).

\section{Rhegmatogenous complications}

Iatrogenic retinal breaks were reported in 29 eyes (4.99\%); all were treated intraoperatively either with endolaser or cryopexy. Of these, 18 (62.1\%) were 20-gauge cases; $10(34.5 \%)$ were 23-gauge cases; 1 (3.4\%) was a 25-gauge case. No patient developed retinal breaks among 27-gauge cases. Retinal breaks tended to occur more frequently as the gauge increased $(p<0.001)$. Twenty-five eyes $(86.2 \%)$ complicated with a retinal break had complete vitrectomy; three eyes (10.3\%) subtotal vitrectomy; and one core vitrectomy. Complete vitrectomy was associated with a higher incidence of retinal breaks than more limited vitrectomy $(p<0.001)$. Cut rates $<1500 \mathrm{cpm}$ were associated with a higher incidence of retinal breaks than higher speeds (89.7 vs. $1.6 \%, p<0.001)$. Phakic eyes $(7.2 \%)$ had a significantly higher incidence of retinal breaks than pseudophakic eyes $(3.2 \%)(p=0.03)$. Of the 19 phakic eyes that had iatrogenic retinal breaks, $15(79 \%)$ had combined phacoemulsification. Retinal breaks tended to occur more frequently when combined phacovitrectomy was 
Table 2 Multivariate regression analyses of potential risk factors for the development of iatrogenic retinal breaks, postoperative retinal detachment and cataract.

\begin{tabular}{|c|c|c|c|c|c|c|c|}
\hline & \multirow[b]{2}{*}{$\begin{array}{l}\text { Categorical (C) (referent category first) or } \\
\text { scaled (S) }\end{array}$} & \multicolumn{2}{|c|}{ Retinal break formation } & \multicolumn{2}{|l|}{$\begin{array}{l}\text { Postoperative RD } \\
\text { development }\end{array}$} & \multicolumn{2}{|l|}{ Cataract formation } \\
\hline & & OR (\%95 CI) & $p$ value & OR (\%95 CI) & $p$ value & OR (\%95 CI) & $p$ value \\
\hline Patient age $^{\mathrm{a}}$ & $\mathrm{S}$ (years) & $1.02(0.98-1.06)$ & 0.273 & $0.94(0.88-1)$ & 0.060 & $0.98(0.96-0.99)$ & 0.044 \\
\hline Patient sex ${ }^{a}$ & C (female, male) & $2.74(1.07-7.03)$ & 0.036 & $1.54(0.31-7.66)$ & 0.598 & $1.63(0.99-2.68)$ & 0.054 \\
\hline Refractive status & C (myopia, hyperopia) & NA & & $0.54(0.17-1.67)$ & 0.286 & NA & \\
\hline Lens status & C (phakic, pseudophakic) & $1.16(0.26-5.19)$ & 0.848 & $1.92(0.26-14.38)$ & 0.525 & NA & \\
\hline \multirow[t]{2}{*}{ Quality of vitrectomy } & C (complete, core, subtotal) & $0.05(0.01-0.46)$ & 0.009 & NA & & $1.60(0.74-3.45)$ & 0.230 \\
\hline & & $0.23(0.05-1.06)$ & 0.059 & & & $0.78(0.39-1.55)$ & 0.476 \\
\hline \multirow[t]{2}{*}{ Gauge } & $\mathrm{S}(20,23,25$ or smaller $)$ & $2.13(0.70-6.46)$ & 0.181 & $0.34(0.05-2.42)$ & 0.281 & $0.66(0.28-1.52)$ & 0.329 \\
\hline & & $0.27(0.02-3.38)$ & 0.313 & $0.09(0.004-1.65)$ & 0.103 & $1.1(0.45-2.61)$ & 0.855 \\
\hline \multirow[t]{2}{*}{ Cutting speed } & $\mathrm{S}(<1500,1500-4000,>4000)$ & $0.03(0.003-0.29)$ & 0.002 & $0.15(0.01-2.33)$ & 0.176 & $1.98(0.97-4.07)$ & 0.063 \\
\hline & & $0.12(0.02-0.76)$ & 0.025 & $1.9(0.19-18.5)$ & 0.578 & $1.34(0.54-3.33)$ & 0.526 \\
\hline $\begin{array}{l}\text { Combined cataract } \\
\text { extraction }\end{array}$ & $\mathrm{C}$ (absent, present) & $0.90(0.19-4.19)$ & 0.898 & NA & & NA & \\
\hline Intraoperative complication & $\mathrm{C}$ (absent, present) & NA & & $1.91(0.34-10.7)$ & 0.460 & NA & \\
\hline
\end{tabular}

$O R$ odds ratio, $C I$ confidence interval, $N A$ not applicable to be included in the regression model.

${ }^{\mathrm{a}}$ Forced demographic covariates.

performed $(16.5$ vs. $2.9 \%, p<0.001)$. It is of note that complete vitrectomy had been performed in most of the combined surgeries that had an iatrogenic break; of the 15 eyes, 14 eyes had complete and only 1 eye had subtotal vitrectomy.

RD occurred in 14 (2.4\%) eyes at a median of 3 months post-vitrectomy (range, 1-50 months). Of the seven eyes that developed RD later than 3 months postoperatively, two had cataract surgery 2 months prior to the development of RD. Having a cataract surgery in the postoperative period was not related with the postoperative RD development $(p=0.14)$. Except from one eye that developed RD 50 months postvitrectomy, none had a history of iatrogenic break formation noticed during the surgery. There were $5(4.7 \%)$ RDs in the 20-gauge; 5 (2.5\%) RDs in 23-gauge; 4 (1.5\%) RDs in the 25gauge; and none in the 27-gauge group $(p=0.18)$. RD occurred in six eyes (3.6\%) in which a complete vitrectomy was performed; in seven eyes $(2.4 \%)$ that had subtotal vitrectomy; and in one eye $(0.8 \%)$ that had core vitrectomy $(p=0.29)$. The mean age of the patients with a RD was 57.4 years, which was slightly younger than 63.2 years of the patients who did not develop a $\mathrm{RD}(p=0.04)$.

Multivariable logistic regression analyses were conducted to assess the impact of a number of factors on the likelihood of development of rhegmatogenous complications. Potential risk factors investigated were refractive status, lens status, vitrectomy quality, gauge, cutting speed and combined phacoemulsification. In addition, presence of any intraoperative complication and postoperative cataract surgery were explored as potential risk factors in the postoperative RD development. The factors associated with rhegmatogenous complications at $p$ value $\leq 0.20$ were included in the fitted model, along with the forced covariates (age, sex). Initial screening of the factors is shown in Supplementary Material 1 and the results of the logistic regression in Table 2. Based on the odds ratios, iatrogenic retinal breaks were less likely to occur in eyes where surgery was limited with core vitrectomy compared with those that had complete vitrectomy (OR 0.05 , $p=0.009$ ), and when cut rates of $1500-4000$ or $>4000$ were used rather than $<1500 \mathrm{cpm}$ (OR 0.03, 0.12; $p=0.002,0.03$, respectively). Regarding the postoperative RD development, regression model yielded none of the potential factors as independently significant.

\section{Other complications}

Cystoid macular oedema occurred in eight eyes (1.4\%). A macular pucker developed in 7 (1.2\%) cases, four of which required a second PPV with membrane removal. Three eyes $(0.5 \%)$ had transient vitreous haemorrhage; and one had choroidal haemorrhage. One eye developed chronic hypotony. Thirteen $(2.2 \%)$ eyes developed an IOP rise; four were controlled with short-term medical therapy; nine $(1.5 \%)$ resulted in glaucoma. Four eyes $(0.7 \%)$, in which posterior hyaloid was left intact during the primary surgery, were reported to have PVD at an average of 21 months (range, 1-36 months) post-vitrectomy, which resulted in floaters, requiring repeat vitrectomy. No endophthalmitis case was reported.

\section{Preoperative symptoms and postoperative patient satisfaction}

Preoperative ophthalmoscopic evaluation revealed that most eyes had huge $(22.2 \%)$ or dense vitreous floaters $(61.6 \%)$; 

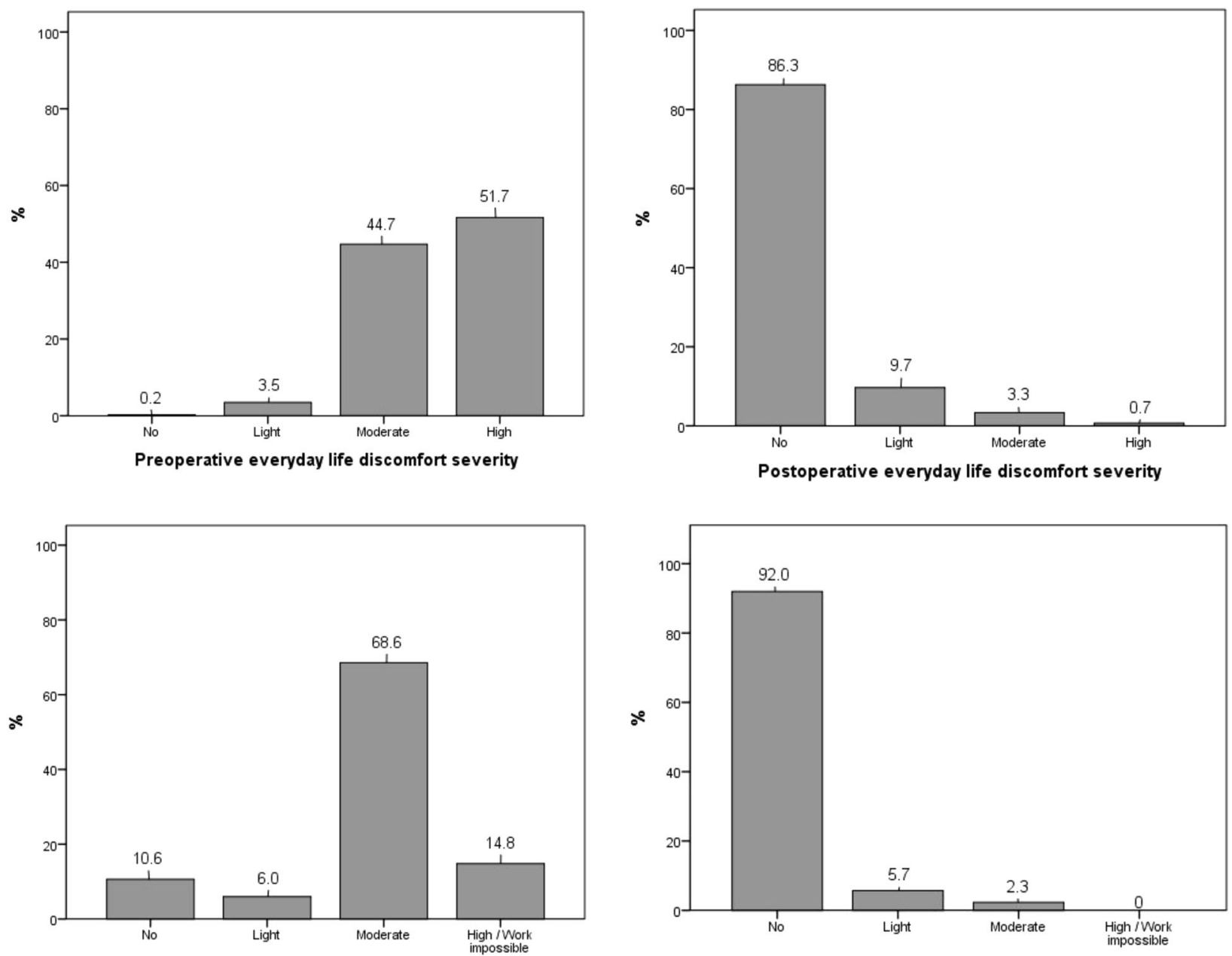

Preoperative professional life discomfort severity

Postoperative professional life discomfort severity
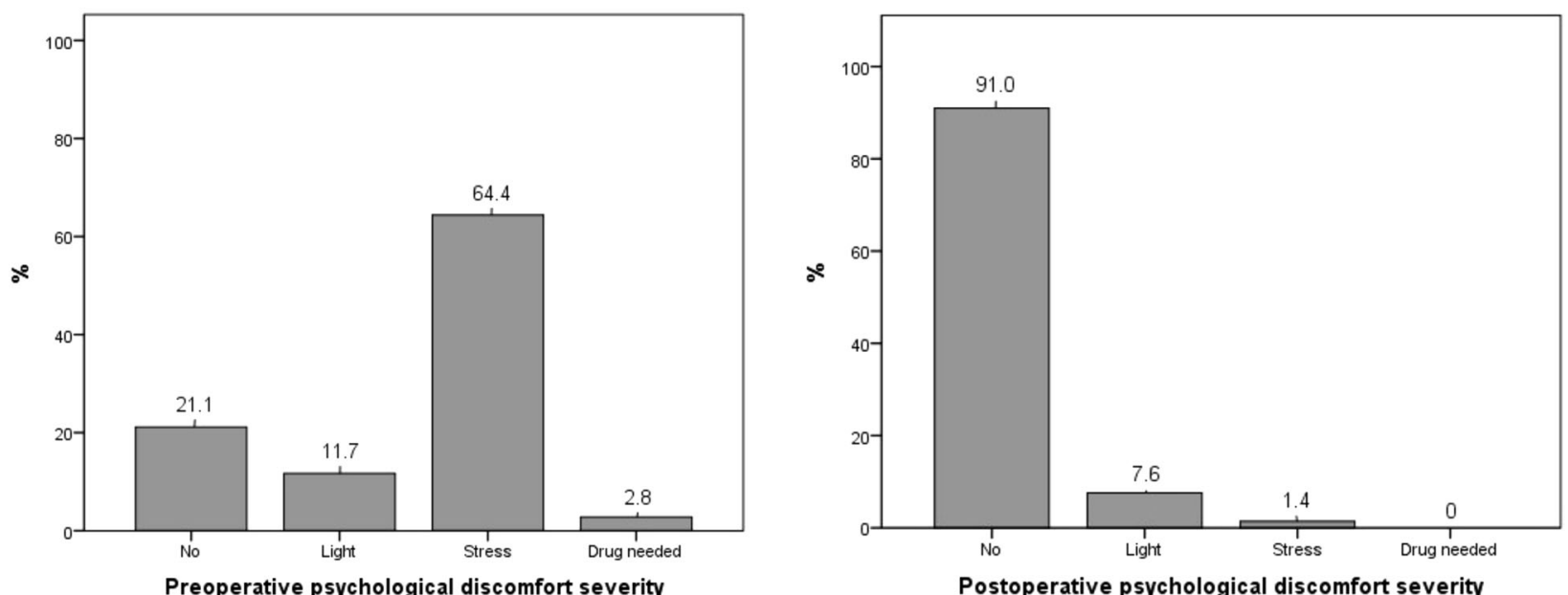

Fig. 1 Influence of floaters on everyday life, professional life and psychological condition of the patients before and after the operation. Preoperative (left column) and postoperative (right column) discomfort levels were reported by $99 \%$ and $98 \%$ of the patients, respectively. After vitrectomy, $86.3 \%$ of the patients were completely cured from their troublesome floaters affecting everyday life (upper row); $92 \%$ of the patients reported complete resolution of symptoms affecting professional life (middle row); and $91 \%$ reported complete resolution of psychological symptoms (lower row). 
whereas a smaller portion had small $(14.6 \%)$ or almost invisible $(1.6 \%)$ floaters. Reported response rate, regarding preoperative and postoperative discomfort levels was $99.1 \%$ and $97.8 \%$, respectively. Most patients $(96.4 \%)$ described the subjective severity of daily symptoms as "moderate" or "high". Floater symptoms diminished significantly after the operation compared with preoperative values $(p<0.001)$. Complete resolution of symptoms affecting everyday life, professional life and psychological condition was reported in 86.3, 92 and 91\%; and only mild symptoms in 9.7, 5.7 and $7.6 \%$ of the cases, respectively (Fig. 1).

Overall satisfaction was reported by 476 patients. Four hundred forty patients $(92.4 \%)$ were "satisfied" or "extremely satisfied" with the outcome. Twenty-six patients (5.5\%) were neutral. Ten patients $(2.1 \%)$ described their symptoms to be "worse" after the surgery. More than half of the dissatisfied cases had complications or persistent floaters.

Multivariable logistic regression analyses were conducted to assess the potential factors that may influence the postoperative symptomatic improvement and satisfaction. Given the sparsity of the observed categories of discomfort, symptomatic improvement was defined as the reduction of the preoperative discomfort from substantial ("moderate/high") to low ("no/light") after the operation. Likewise, since there are few unsatisfied patients, patients were aggregated into: satisfied ("satisfied/extremely satisfied") and not satisfied ("equal/ worse"). Predictors evaluated in the analyses were floater size, duration of symptoms, refractive status, lens status, vitrectomy quality, gauge, cutting speed, combined phacoemulsification and presence of any intraoperative or postoperative complications (Supplementary Material 2). We included in the fitted regression model, the factors to be independently associated with symptomatic improvement and satisfaction at $p$ value $\leq 0.20$, along with the forced covariates (age and sex). Regression analysis showed that the patients with almost invisible to small preoperative floaters were 2.5 times more likely not to be satisfied with the postoperative results than the patients with dense or huge floaters $(p=0.02)$. Regarding symptomatic improvement, patients with postoperative complications were found to be less likely to report improvement in their symptoms affecting everyday life and professional life (OR 0.31, $p=0.01$ and OR 0.15, $p=0.02$ ) (Table 3).

\section{Discussion}

While there remains the debate among vitreoretinal surgeons whether the symptoms of patients justify the potential risks of a surgery, a significant number of patients demand getting rid of bothersome floaters [5-7]. The present large-scale multicentre study therefore sought to determine the outcomes of vitrectomy and identify possible risk factors responsible for the complications.

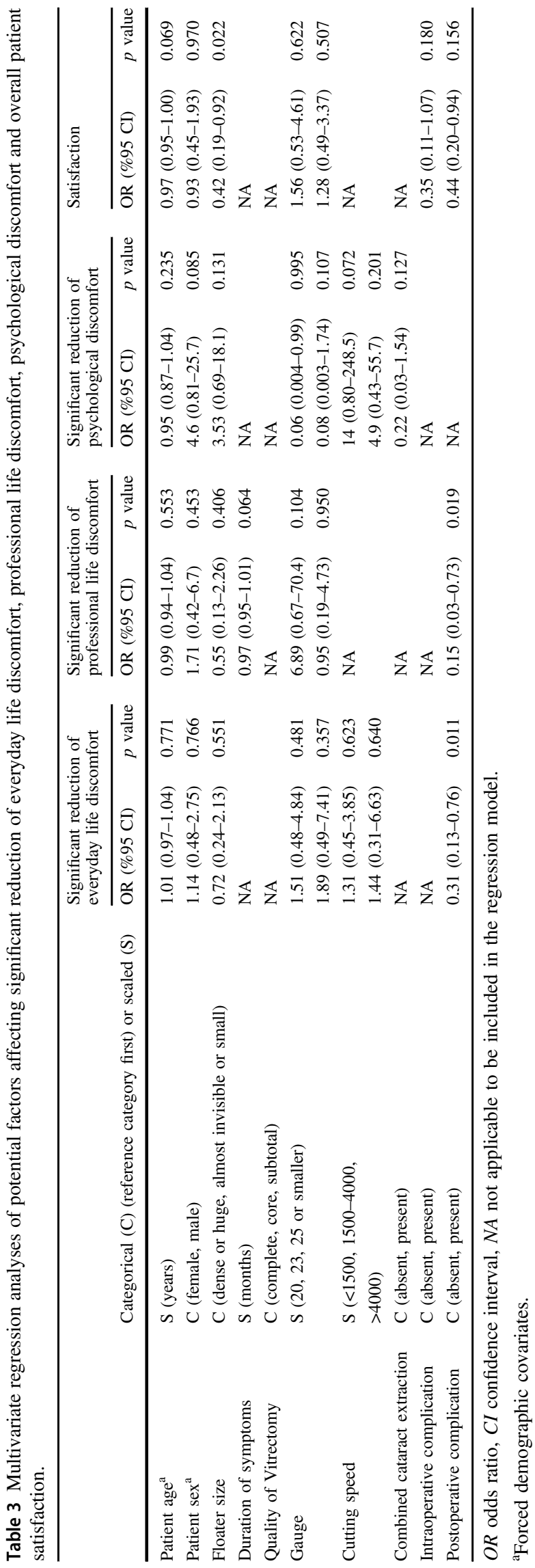


Given the invasive nature of the PPV, potential drawbacks in these patients are rhegmatogenous complications and cataract formation. Reported rates of iatrogenic retinal breaks during vitrectomy for other elective indications (e.g. macular hole/pucker) vary between 11 and $24 \%$ for 20 -gauge systems $[13,14]$, and between 3 and $16 \%$ for smaller-gauge procedures [14-16]. The overall rate of 5\% in this study falls in the lower end of the spectrum. We observed a higher rate of retinal breaks with 20 gauge $(17 \%)$ compared with smallergauge vitrectomy $(5.4 \%)$; however, this difference failed to reach significance on multivariable analysis. One of the significant risk factors was the extensive vitrectomy with peripheral base shaving and PVD induction. Despite the limitation that the proportion of preexisting PVD was unknown in the complete vitrectomy group, extensive surgery was related with the increased risk of iatrogenic retinal breaks compared with core vitrectomy where no PVD was induced. Other risk factor significantly associated with the retinal break formation was the use of low cutting speeds. Even though no study to date discussed the role of cut rates in the development of iatrogenic breaks during floater vitrectomy, literature reports on other elective macular surgeries suggest that higher speed could reduce the incidence of retinal breaks, by minimizing the vitreous turbulence and dynamic traction $[17,18]$. Multivariable analysis showed that the use of cut rates $\geq 1500$ cpm was safer than $<1500 \mathrm{cpm}$. However, the use of cut rates $>4000 \mathrm{cpm}$ did not differ significantly from the use of $1500-4000 \mathrm{cpm}$.

Several authors proposed minimalistic surgical approaches in an attempt to prevent complications [19, 20]. Sebag et al. [19] reported low complication rates by adopting a more limited surgery, involving core vitrectomy without the posterior hyaloid removal unless there is a preexisting PVD. Of 60 cases, they reported macular pucker formation in one eye and no cases of iatrogenic retinal breaks, postoperative $\mathrm{RD}$, vitreous haemorrhage or endophthalmitis at an average follow-up of 17.5 months. Mason et al. [20] described a similar approach in their series of 168 eyes; with leaving posterior hyaloid in most eyes (85\%), and shaving base only in the presence of retinal breaks. They reported iatrogenic retinal breaks in 12 eyes $(7.1 \%)$, and no postoperative RD at an average follow-up of 18 months. They found no significant association between retinal breaks and PVD induction; however, as the authors also refer to, this may be due to the fact that only a small number of patients underwent PVD induction. Conversely, higher complication rates were reported by Schulz-Key et al. and De Nie et al. in two series with longer follow-ups of 37 and 26.4 months, respectively [6, 10]. Schulz-Key et al. [10] performed mostly core vitrectomy, yet they reported postoperative RD in $6.8 \%$ of eyes. It is noteworthy that $5.5 \%$ of the RD occurred in the long-term, between 2 and 4 years postvitrectomy. A possible explanation for this late incidence may be spontaneous and anomalous PVD development over time since the vitrectomy was limited to central vitreous. In the latter study by De Nie et al. [6], in which patients underwent 20- or 23-gauge vitrectomy with PVD induction, postoperative RD rates were found as high as $10.9 \%$. As the authors commented, lack of routine search with scleral indentation to detect retinal breaks might be responsible for high incidence. In contrast, postoperative RD occurred rather early and infrequent in the present study. We observed RD in $2.4 \%$ of patients at a median of 3 months post-vitrectomy; and none except one case were in eyes where the retinal breaks noted intraoperatively. This may indicate the possibility of unrecognized retinal breaks at the time of surgery, and underscores the importance of searching for intraoperative breaks before concluding the surgery. The 20-month follow-up in this study is longer than the follow-ups in the studies by Sebag et al. [19] and Mason et al. [20], but less than 37-month-follow-up by Schulz-Key et al. [10]. RD may be expected to increase in the longer term due to a postoperative PVD development in eyes where posterior hyaloid was left intact. Another point that draws attention is that two patients developed RD only after having a cataract surgery. It is arguable that the RD in these cases may be related to cataract extraction with consecutive PVD rather than vitrectomy itself. However, this hypothesis was not supported statistically.

Cataract formation was frequent in our series, necessitating phacoemulsification in nearly half the phakic cases $(48.6 \%)$ at an average of 16 months. This was comparable to the rates of $38-50 \%$ given by De Nie et al. [6] and Tan et al.[9] in series where full vitrectomy was performed. Reported rates of cataract formation was lower at 23.5 [19] and $22.5 \%$ [20] in two other studies where sparing of anterior vitreous were advocated to protect the lens against free oxygen radicals. However, the exact pathogenesis of post-vitrectomy cataract formation is not clear and many other factors have been proposed to play a role, including surgery duration, mechanical damage from instrumentation, intraoperative light toxicity, type and amount of irrigating solution and intraocular inflammation $[21,22]$. We failed to find any significant difference among the eyes to whom more limited or extensive surgery were performed; however, employment of heterogenous surgical approaches by different surgeons may be expected in this study and could affect the outcome.

Visual acuity in floaters is generally minimally affected and does not correlate with the symptoms. Therefore, patient selection as well as efficacy assessment have been addressed by evaluating the difficulty of performing daily tasks, the impact of the symptoms on patients' lives, or patient satisfaction $[6,8,10]$. We separately assessed the symptoms affecting different aspects of life and overall satisfaction after surgery. Most patients stated that they 
had "moderate" to "high" level of discomfort before surgery; and complete resolution of the symptoms affecting everyday life, professional life and psychological condition was reported in about $90 \%$. Overall, $92.4 \%$ of the patients were satisfied with the outcome. This is in accordance with earlier studies, reporting high patient satisfaction post-surgery, ranging from 88 to $96 \%$ $[8,10,20]$. The investigation of the potential factors that may negatively influence the symptomatic improvement and patient satisfaction yielded two significant results. The first one was the presence of complications as expected. However, the second one was more noteworthy. Patients with almost invisible/small preoperative vitreous opacities were more likely not to be satisfied with the results than the patients with dense/huge opacities. This indicates the possibility that psychological factors might be related with the discomfort severity. Some authors have shown that certain personality traits may be related with an increased awareness and perceived discomfort with floaters [23-25]. A recent study found a higher incidence of psychological problems, such as depression, anxiety and stress among symptomatic floater patients compared with the asymptomatic controls with vitreous opacities. In addition, the degree of floater-related discomfort and severity of the psychological distress was correlated [24]. The proportion of the patients reporting persistent symptoms post-treatment that affect their psychological condition was not significantly different between the groups with almost invisible/small preoperative vitreous opacities and with huge/dense opacities (3.3 vs. $1.1 \%, p=0.13$ ). However, the dissatisfaction rate was significantly higher in the patients presenting with smaller opacities. These results raise the concern whether the severity of symptoms constitute enough of an indication especially when there is no corresponding opacity detected ophthalmoscopically. Several methods, including contrast sensitivity, straylight measurements, quantitative ultrasound and optical coherence tomography infrared video scans, have been proposed to define the target group more objectively $[19,26,27]$. Validation of these tests in large-scale prospective studies and better assessment of patients' impairment level and expectations may help to reduce the concerns.

Limitations of this study include the retrospective design and evaluations based on self-reporting; which may both cause selection bias and affect the quality of the results. In addition, detailed information regarding preoperative PVD status was not available in all cases. Despite these pitfalls, considering that a large number of surgeons from 16 countries contributed to the study, the outcomes reported here are expected to be representative of a wide population globally and largely applicable.
In conclusion, this large-scale multicentre study demonstrated that the PPV was effective in alleviating the symptoms of floaters yet it is an invasive approach with risk of complications. Performing vitrectomy limited to central vitreous only and using cutting speeds $>1500 \mathrm{cpm}$ may increase the safety. The degree of impairment must be well understood, especially in patients whose distress level is out of proportion with the severity of the opacities. Further studies that may elucidate objective parameters to support the indication for surgery are thus warranted.

\section{Summary}

\section{What was known before}

- Vitrectomy for floaters has remained controversial among vitreoretinal surgeons.

- The reported complication rates of floater vitrectomy vary substantially and still lack sufficient peer-reviewed evidence in large series.

- The effect of vitrectomy parameters on safety in floater vitrectomy has not been investigated.

\section{What this study adds}

- Providing data on 581 cases from 16 countries undergoing floater vitrectomy, this study may be expected to be representative of a wide population globally.

- Hence, the success as well as the complication rates may be largely applicable and guide counselling of patients on the efficacy and safety profiles of this intervention.

- Vitrectomy parameters such as core vitrectomy and the use of cut rates above 1500 cuts/min have been found to increase the safety of the procedure.

\section{Compliance with ethical standards}

Conflict of interest The authors declare that they have no conflict of interest.

Publisher's note Springer Nature remains neutral with regard to jurisdictional claims in published maps and institutional affiliations.

\section{References}

1. Tozer K, Johnson M, Sebag J. Vitreous aging and posterior vitreous detachment. In: Sebag J, editors. Vitreous-in health and disease. New York: Springer-Verlag; 2014. p. 131-50.

2. Gale J, Ikuno Y. Myopic vitreopathy. In: Sebag J, editors. Vitreous—in health and disease. New York: Springer-Verlag; 2014. p. 113-29. 
3. Roufail ED, Polkinghorne P. Vitreous floaters. Compr Ophthalmol Update. 2006;7:171-7.

4. Henry CR, Smiddy WE, Flynn HW Jr. Pars plana vitrectomy for vitreous floaters: is there such a thing as minimally invasive vitreoretinal surgery? Retina. 2014;34:1043-5.

5. Wagle AM, Lim WY, Yap TP, Neelam K, Au Eong KG. Utility values associated with vitreous floaters. Am J Ophthalmol. 2011;152:60-5 e1.

6. de Nie KF, Crama N, Tilanus MA, Klevering BJ, Boon CJ. Pars plana vitrectomy for disturbing primary vitreous floaters: clinical outcome and patient satisfaction. Graefes Arch Clin Exp Ophthalmol. 2013;251:1373-82.

7. Zou H, Liu H, Xu X, Zhang X. The impact of persistent visually disabling vitreous floaters on health status utility values. Qual Life Res. 2013;22:1507-14.

8. Delaney YM, Oyinloye A, Benjamin L. Nd:YAG vitreolysis and pars plana vitrectomy: surgical treatment for vitreous floaters. Eye (Lond). 2002;16:21-6.

9. Tan HS, Mura M, Lesnik Oberstein SY, Bijl HM. Safety of vitrectomy for floaters. Am J Ophthalmol. 2011;151:995-8.

10. Schulz-Key S, Carlsson JO, Crafoord S. Longterm follow-up of pars plana vitrectomy for vitreous floaters: complications, outcomes and patient satisfaction. Acta Ophthalmol. 2011;89:159-65.

11. Tsai WF, Chen YC, Su CY. Treatment of vitreous floaters with neodymium YAG laser. Br J Ophthalmol. 1993;77:485-8.

12. Little HL, Jack RL. Q switched neodymium: YAG laser surgery of the vitreous. Graefes Arch Clin Exp Ophthalmol. 1986; 224:240-6.

13. Moore JK, Kitchens JW, Smiddy WE, Mavrofrides EC, Gregorio G. Retinal breaks observed during pars plana vitrectomy. Am J Ophthalmol. 2007;144:32-6.

14. Covert DJ, Henry CR, Bhatia SK, Croskrey J, Sanchez CR, Han DP. Intraoperative retinal tear formation and postoperative rhegmatogenous retinal detachment in transconjunctival cannulated vitrectomy systems compared with the standard 20-gauge system. Arch Ophthalmol. 2012;130:186-9.

15. Tan HS, Mura M, de Smet MD. Iatrogenic retinal breaks in 25gauge macular surgery. Am J Ophthalmol. 2009;148:427-30.

16. Hikichi $\mathrm{T}$, Kosaka $\mathrm{S}$, Takami $\mathrm{K}$, et al. Incidence of retinal breaks in eyes undergoing 23 -gauge or 20-gauge vitrectomy with induction of posterior vitreous detachment. Retina. 2012;32: $1100-5$.

17. Mura M, Barca F, Dell'Omo R, Nasini F, Peiretti E. Iatrogenic retinal breaks in ultrahigh-speed 25-gauge vitrectomy: a prospective study of elective cases. Br J Ophthalmol. 2016;100: 1383-7.

18. Rizzo S, Genovesi-Ebert F, Belting C. Comparative study between a standard 25-gauge vitrectomy system and a new ultrahigh-speed 25-gauge system with duty cycle control in the treatment of various vitreoretinal diseases. Retina. 2011;31: 2007-13.

19. Sebag J, Yee KM, Wa CA, Huang LC, Sadun AA. Vitrectomy for floaters: prospective efficacy analyses and retrospective safety profile. Retina. 2014;34:1062-8.

20. Mason JO, Neimkin MG. Safety, efficacy, and quality of life following sutureless vitrectomy for symptomatic vitreous floaters. Retina. 2014;34:1055-61.

21. Ling CA, Weiter JJ, Buzney SM, Lashkari K. Competing theories of cataractogenesis after pars plana vitrectomy and the nutrient theory of cataractogenesis: a function of altered aqueous fluid dynamics. Int Ophthalmol Clin. 2005;45:173-98.

22. Cheng L, Azen SP, El-Bradey MH, et al. Duration of vitrectomy and postoperative cataract in the vitrectomy for macular hole study. Am J Ophthalmol. 2001;132:881-7.

23. Schiff WM, Chang S, Mandava N, Barile GR. Pars plana vitrectomy for persistent, visually significant vitreous opacities. Retina. 2000;20:591-6.

24. Kim YK, Moon SY, Yim KM, Seong SJ, Hwang JY, Park SP. Psychological distress in patients with symptomatic vitreous floaters. J Ophthalmol. 2017;2017:3191576.

25. Cipolletta S, Beccarello A, Galan A. A psychological perspective of eye floaters. Qual Health Res. 2012;22:1547-58.

26. Mura M, Engelbrecht LA, de Smet MD, Papadaki TG, van den Berg TJ, Tan HS. Surgery for floaters. Ophthalmology. 2011;118:1894-e1.

27. Shah CP, Heier JS. YAG laser vitreolysis vs sham YAG vitreolysis for symptomatic vitreous floaters: a randomized clinical trial. JAMA Ophthalmol. 2017;135:918-23.

\title{
for the EVRS Floaters Study Group
}

\begin{abstract}
Ahmed B. Sallam ${ }^{10} \cdot$ Abdallah A. Ellabban $^{11} \cdot$ Barbara Parolini $^{12} \cdot$ Sengul Ozdek $^{13} \cdot$ Jay Chhablani $^{14} \cdot$ Ivan Fiser $^{15}$. Filip Brazda ${ }^{15} \cdot$ Petr Bedrich $^{15} \cdot$ Robert Kanovsky $^{15} \cdot$ Philippe Koch $^{16} \cdot$ Erkan Unsal $^{17} \cdot$ Ferenc Kuhn $^{18}$. Frédéric Matonti ${ }^{19} \cdot$ Giampaolo Gini $^{20} \cdot$ Petros Aristodemou $^{21} \cdot$ Camiel J. F. Boon $^{22} \cdot$ Zsuzsanna Szijarto $^{23}$. Gérard Estacy ${ }^{24}$. Christine Kusserow ${ }^{25}$. Isabella Turco ${ }^{26}$. Idriss Badat ${ }^{27}$. Silvia Bopp ${ }^{28}$ - Alexandra Brix ${ }^{29}$. Klaus Lucke ${ }^{30}$ - Andreas Schüler ${ }^{30}$ - Didier Ducournau ${ }^{31} \cdot$ Miroslav Veith $^{32} \cdot$ Vito Primavera $^{33}$ - Nicola Delle Noci ${ }^{34}$. Frédéric Hamon ${ }^{35} \cdot$ Ron A. Adelman ${ }^{36} \cdot$ Roberto Uy $^{37} \cdot$ Rita Soyeur $^{38} \cdot$ Eric Fourmaux $^{39}$. Constantinos Demosthenos ${ }^{40}$. Frank Becquet ${ }^{27}$ - Jean-Paul Berrod ${ }^{41} \cdot$ Isabelle Hubert $^{42} \cdot$ Stepan Rusnak $^{43}$. Pedro Neves ${ }^{44}$. David Martins ${ }^{45}$. Gian Marco Tosi ${ }^{46} \cdot$ Agnès Glacet-Bernard $^{47} \cdot$ Benedetto Mochi $^{48}$. Pedro Romero Aroca ${ }^{49,50} \cdot$ Pierre-Olivier Lafontaine ${ }^{51} \cdot$ Lyubomyr Lytvynchuk $^{52} \cdot$ Manzar Saeed $^{53} \cdot$ Manish Gunda $^{53}$. Charles Gremillion ${ }^{54}$
\end{abstract}

10 Jones Eye Institute, University of Arkansas for Medical Sciences, Little Rock, AR, USA

11 Hull University Teaching Hospitals NHS Trust, Hull, UK

12 EyeCare Clinic, Via Cefalonia 70, 25124 Brescia, Italy
13 Gazi University School of Medicine, 06500 Ankara, Turkey

14 Smt. Kanuri Santhamma Centre for Vitreo-Retinal Diseases, L V Prasad Eye Institute, Hyderabad, India 
15

Lexum European Eye Clinic, Antala Staska 1670/80, Prague 4, Czech Republic

16 Cabinet Ophtalmologique, Avenue Paul Hymans 126, 1200 Woluwe-St-Lambert, Belgium

17 Department of Ophthalmology, Istanbul Research and Education Hospital, Istanbul, Turkey

18 Helen Keller Foundation for Research and Education, Birmingham, AL, USA

Department of Ophthalmology, Nord Hospital, Aix Marseille University, Institut de Neurosciences de la Timone, Marseille, France

Eye Unit, Southampton University Hospital, Southampton, UK Bristol, UK

Department of Ophthalmology, Leiden University Medica Center, J3-S, Albinusdreef 2, 2333 ZA Leiden, the Netherlands

University pécs, Nyar u. 8., 7624 Pécs, Hungary

Capio Augenklinik Universitätsallee, Parkallee 301, D-28213 Bremen, Germany

niversitätsallee, Parkallee 301, D-28213

Bremen, Germany

31 Department of Retinal Surgery, Clinique Sourdille Nantes, SaintHerblain, France

32 Department of Ophthalmology, University Hospital Kralovske Vinohrady and Third Faculty of Medicine, Charles University in Prague, Prague, Czech Republic

Department of Ophthalmology, ASL BA-Ospedale della Murgia "Fabio Perinei,", Altamura, Italy

34 Department of Ophthalmology, Policlinico di Foggia, University of Foggia, Foggia, Italy
35 Centre Helios Ophtalmologie, 8 Rue de la Ferme Dai Baita, 64500 Saint-Jean-de-Luz, France

36 Yale University School of Medicine, New Haven, CT, USA

37 Retina Consultant PerfectSight NCR - National Capital Region, Quezon City, Philippines

388 Boulevard Hauterive, 64075 Pau, France

3986 Rue Palais Gallien, 33000 Bordeaux, France

40 The Queen Elizabeth Hospital, King's Lynn NHS Foundation Trust, Gayton Road, King's Lynn PE30 4ET, UK

41 Department of Ophthalmology, Centre Hospitalier Universitaire de Nancy, Nancy, France

42 Department of Ophthalmology, Nancy University Hospital, Nancy, France

43 FN Plzen Instutite, Alej Svobody 80, Plzen, Czech Republic

44 Hospital Sao Bernardo, R. Camilo Castelo Branco, 2910-446 Setúbal, Portugal

45 Centro Hospitalar de Setubal,E.P.E., Setubal, Portugal

46 University of Siena, Via Aldo Moro 2, 53100 Siena, Italy

47 Department of Ophthalmology, Centre Hospitalier Intercommunal de Créteil, Université Paris-Est-Créteil, Paris 12 University, Créteil, France

48 University of Genoa, Piazza della Vittoria, 14/1 c/o Is.O.L.A, 16121 Genoa, Italy

49 Ophthalmology Service, University Hospital Sant Joan, Reus, Spain

50 Institut de Investigacio Sanitaria Pere Virgili (IISPV), Universitat Rovira and Virgili, Reus, Spain

51 Centre Ophtalmologique OCULUS, 124 Allée Albert Sylvestre, Chambery, France

52 Department of Ophthalmology, Justus-Liebig-University Giessen, Eye Clinic, University Hospital Giessen and Marburg $\mathrm{GmbH}$, Giessen, Germany

53 Queen Elizabeth Hospital, Gayton Road, King's Lynn, UK

54 Englewood Community Hospital, 2400S McCall Rd A, Englewood, FL 34224, USA 